\title{
Evaluación de los estilos de enseñanza universitaria en el contexto virtual
}

Evaluation of university teaching styles in the virtual context

\author{
Edith Soledad Olivera Carhuaz ${ }^{\square}$, (iD Daniel Edgardo Yupanqui Lorenzo
}

Universidad Inca Garcilaso de la Vega. Lima, Perú

Recibido: $28 / 06 / 2020 \quad$ Revisado: $29 / 08 / 2020 \quad$ Aceptado: 09/11/2020 Publicado: 16/12/2020

\section{RESUMEN}

La situación que se vive a nivel mundial ha impulsado la educación virtual, de modo que los docentes universitarios se han visto obligados a adaptarse a esta forma exclusiva de enseñar, por ende, surgen cuestionamientos sobre la didáctica, metodología y recursos que emplean en este entorno para lograr que los estudiantes aprendan. Dado este fenómeno, el objetivo del presente estudio fue conocer los estilos de enseñanza predominantes en los docentes universitarios de una institución privada de Lima. La metodología fue descriptiva de enfoque cuantitativo y la muestra estuvo conformada por 114 docentes de la facultad de Hotelería y Turismo de la institución en mención. Los resultados obtenidos determinaron que el estilo de enseñanza que predomina es funcional, seguido del formal y estructural. Estos estilos se relacionan con el conocimiento académico de docencia universitaria. Palabras clave: Didáctica, enseñanza, virtual, estilos, pandemia, aprendizaje.

\section{ABSTRACT}

The situation that is lived worldwide has promoted virtual education, so that university teachers have been forced to adapt to this exclusive way of teaching, therefore, questions arise about the didactics, methodology and resources they use in this environment to get students to learn. Given this phenomenon, the objective of the present study was to find out the predominant teaching styles among university teachers in a private institution in Lima. The methodology was descriptive of a quantitative approach and the sample was made up of 114 teachers from the Hospitality and Tourism faculty of the mentioned institution. The results obtained determined that the prevailing teaching style is functional, followed by formal and structural. These styles are related to the academic knowledge of university teaching. Keywords: Didactics, teaching, virtual, styles, pandemic, learning. 


\section{INTRODUCCIÓN}

La pandemia del Covid-19, ha exigido que la educación dé un giro de $360^{\circ}$, debido a que las clases presenciales en la mayoría de países se han restringido, por lo tanto, se ha tenido que migrar a entornos virtuales, es así que la tecnología y la revolución digital han impulsado diferentes propuestas educativas en línea (Gómez, 2017).

En nuestro país, las universidades, han implementado plataformas digitales para cumplir con su propósito y dar continuidad al periodo académico, garantizando la accesibilidad, adaptabilidad y calidad educativa, así como la satisfacción del estudiante, según lo establecido en la Resolución Nº39, emitida por la Sunedu.

En ese sentido, la capacitación al personal docente ha priorizado el manejo de los recursos en línea y poco o nada la metodología, las estrategias de enseñanza o las formas de evaluar en esta nueva realidad, por lo que surgen una serie de interrogantes relacionadas a la práctica docente que involucra el estilo de enseñar que prioriza el maestro. Gómez (2017) afirma que: "Las actuales plataformas digitales y los entornos virtuales de aprendizaje están implicando nuevos modelos de enseñanza, puesto que ofrecen recursos didácticos innovadores de los que no existían antecedentes." (p. 61).

Es conveniente aclarar que la enseñanza engloba un método, dentro de la cual se ven inmersos diferentes elementos como la técnica, los estilos y las estrategias; además de ser un constructo mediador entre el docente, el estudiante y el contenido (Lebrero-Casanova, Almagro \& Sáenz-López, 2019). De modo que, el método de enseñanza se refiere, de manera general, a la forma de conducir el proceso; mientras que, de forma específica, se define como un accionar mediante el cual el docente reflexiona, se adapta, y contextualiza su práctica, teniendo en cuenta el contenido, los objetivos y el procedimiento individual del estudiante (Sicilia \& Delgado, 2002).

Los estilos de enseñanzas son consecuencia de los estilos de aprendizaje, es decir, que la primera involucra una respuesta del estudiante ante una determinada asignatura, la cual propone y dispone de técnicas pedagógicas para lograr la aprehensión de conocimientos, basándose en los estilos en los que el educando aprende. (Renés \& Martínez, 2016; Rendón, 2013; De León, 2006). Si bien, ambas definiciones reflejan un mismo sentido, el concepto de estilos de enseñanza no han variado. Es así que Delgado (1996), refiere que es la forma que toma un docente para efectuar la interacción con su alumno, en función del conocimiento que se desea transmitir. González-Peiteado (2013) aduce que “...un docente no puede abordar con eficacia la tarea de optimizar su desempeño en el aula, si no atiende las peculiaridades del alumno." (p. 52). Por ende, se requiere que los profesionales que dirigen estos procesos tengan conocimientos académicos respecto a didáctica universitaria, (Aguilera, 2012). Condición que se vuelve una exigencia hoy en día, donde las formas de comunicación han cambiado, dejando de lado el contacto directo. (Arias, Morales, Meroño \& Calderón, 2019; Rodríguez, Delgadillo \& Torres, 2018).

Para el análisis, se considera la conceptualización de Martínez (2009), quien propone cuatro tipos de estilos, cuyas diferencias están referidas a la interacción y contacto con el estudiante.

El estilo abierto involucra el dinamismo del docente. Generalmente, se prioriza el trabajo en equipo, garantizando la espontaneidad y la generación de ideas de los estudiantes de 
acuerdo a las actividades propuestas en base a situaciones reales, teniendo en cuenta la flexibilidad de lo programado (Martínez, 2009). Este estilo suele relacionarse con el aprendizaje activo (Chiang, Díaz, Rivas \& Martínez, 2013).

Por otra parte, el estilo formal se caracteriza por que los docentes planifican sus lecciones con anticipación y cumplen a cabalidad todo lo programado (Renés et al., 2013). Además, mantienen una comunicación efectiva y buscan, en todo momento, la reflexión individual del estudiante. (Martínez, 2009).

Asimismo, el estilo estructurado privilegia la planificación de las clases; sin embargo, da mayor énfasis al marco teórico que a los trabajos prácticos en equipo, de forma que los ejercicios individuales propuestos se acompañan de la forma de resolución paso a paso (Renés et al., 2013). Potencian con mayor frecuencia las propuestas intelectuales antes que las afectivas, por ende, toda actividad debe mantener un propósito claro y objetivo (Martínez, 2009).

Por el contrario, el estilo funcional se caracteriza porque los docentes planifican sus actividades pedagógicas en relación a la viabilidad y concreción de objetivos. Buscan que el estudiante aprenda por medio de la práctica y experimentación a través del trabajo en equipo (Chiang et al., 2013; Renés et al., 2013). Suelen llevar a expertos a clase, para que los estudiantes entiendan y aprendan de ellos. Valoran el resultado más que el proceso; y buscan que el estudiante no fracase. Muestran afectividad por los estudiantes durante las sesiones de aprendizaje (Martínez, 2009).

Todo estilo de enseñanza está dirigido a lograr el aprendizaje. Se trata de comprender al estudiante, de adaptarse a la materia o tema, y generar un estilo que convenga tanto al docente como al estudiante. De modo que, la investigación busca conocer los estilos de enseñanza predominante dentro del contexto virtual debido a la pandemia mundial.

\section{MATERIALES Y MÉTODOS}

El cuestionario referente a estilos de enseñanza fue desarrollado por Chiang et al. (2013). Ha sido adaptado y cuenta con evidencia de validez y confiabilidad. Se compone por cinco estilos de enseñanza: activo, formal, estructurado y funcional, así mismo, comprende 71 ítems con respuestas dicotómica. Para el presente estudio se procedió a obtener el análisis de confiabilidad mediante el Alfa de Cronbach el cual fue de 0,70; dicho coeficiente es alto, lo cual permite hacer uso del instrumento. Por otro lado, se realizó un análisis de correlación factor-test, y se determinó que todas las dimensiones mantenían correlación positiva estadísticamente significativa.

El enfoque es cuantitativo, de tipo básica y sigue un diseño descriptivo; no experimental y de corte transversal (Hernández-Sampieri \& Mendoza, 2018). El proceso de recolección de datos se desarrolló con rigurosidad, respetando las normas éticas de consentimiento informado y confidencialidad. Asimismo, se tuvo el análisis de la calidad de los datos obtenidos para el procesamiento y sistematización de los resultados mediante el empleo del paquete estadístico SPSS v. 25.

La muestra estuvo constituida por 114 docentes de una universidad privada de Lima, Perú, licenciada por la Sunedu. Dichos docentes pertenecen a la facultad de Hotelería y Turismo, entre hombres y mujeres, con el grado mínimo de maestría y de diferentes especialidades, Asimismo, en todo momento se tuvo en cuenta los criterios excluyentes para mayor rigurosidad de selección. 
RESULTADOS Y DISCUSIÓN

Se desarrollaron procesos estadísticos descriptivos y de frecuencia para el análisis de los datos. De modo que, el conocer la muestra y efectuar relaciones cualitativas amplían la visualización de los estilos de enseñanza que priorizan los participantes.

Tabla 1. Conocimiento académico de docencia universitaria.

\begin{tabular}{lcc} 
Respuesta & Frecuencia & Porcentaje \\
\hline No & 31 & $27 \%$ \\
Sí & 83 & $73 \%$ \\
Total & 114 & $100 \%$ \\
\hline
\end{tabular}

Fuente. Elaboración propia

En la tabla 1, ante la pregunta si el docente cuenta con estudios o especializaciones en docencia o enseñanza universitaria, el $27 \%$ respondió que no, mientras que el $73 \%$ adujo que sí. Cabe precisar, que el conocimiento teórico de los métodos, estrategias y técnicas de enseñanza permite un mejor desempeño del docente.

Tabla 2. Frecuencia de los niveles en los estilos de enseñanza

\begin{tabular}{lcccccccc}
\hline \multirow{2}{*}{ Niveles } & \multicolumn{2}{c}{ Abierto } & \multicolumn{2}{c}{ Formal } & \multicolumn{2}{c}{ Estructurado } & \multicolumn{2}{c}{ Funcional } \\
\cline { 2 - 10 } & $\mathrm{F}$ & $\%$ & $\mathrm{~F}$ & $\%$ & $\mathrm{f}$ & $\%$ & $\mathrm{~F}$ & $\%$ \\
\hline Muy baja & 24 & $21,1 \%$ & 5 & $4,4 \%$ & 17 & $14,9 \%$ & 6 & $5,3 \%$ \\
Baja & 56 & $49,1 \%$ & 26 & $22,8 \%$ & 25 & $21,9 \%$ & 9 & $7,9 \%$ \\
Moderada & 12 & $10,5 \%$ & 46 & $40,4 \%$ & 35 & $30,7 \%$ & 54 & $47,4 \%$ \\
Alta & 14 & $12,3 \%$ & 29 & $25,4 \%$ & 20 & $17,5 \%$ & 26 & $22,8 \%$ \\
Muy alta & 8 & $7,0 \%$ & 8 & $7,0 \%$ & 17 & $14,9 \%$ & 19 & $16,7 \%$ \\
Total & 114 & $100,0 \%$ & 114 & $100,0 \%$ & 114 & $100,0 \%$ & 114 & $100,0 \%$ \\
\hline Media & 30,62 & 29,71 & 30,19 & 29,96 \\
D.E. & 2,02 & & 1.82 & 2.37 & \multicolumn{2}{c}{1,67}
\end{tabular}

Fuente. Elaboración propia
Dentro del esquema de enseñanza se marcan cuatro estilos principales para este estudio: abierto, formal, estructurado y funcional. En la tabla 2, se aprecian los puntajes obtenidos en los diferentes estilos de enseñanza, siendo el predominante el estilo funcional con un nivel medio de $47,4 \%$ y los niveles alta y muy alta suman un $39,5 \%$ de la muestra, seguido del estilo formal y estructurada, y finalmente, el estilo abierto cuya predominancia es bajo y muy bajo $(49,1 \%$ y $21,1 \%$, respectivamente). Por otro lado, se presentan las medias y desviación estándar de las puntuaciones.

Entre todos los estilos, el abierto se caracteriza por ser parte de docentes que promueven el trabajo cooperativo, la generación de nuevas ideas y generalmente cambian constantemente su metodología pedagógica (Collantes-Hidalgo, 2016); de modo que se suele genera un vínculo de aprendizaje con estudiantes de tipo reflexivo, basado en un enfoque constructivista (Martínez, 2009). A diferencia del estudio de Chiang et al. (2013) el estilo abierto mantuvo niveles bajos de presencia en los docentes; además, un estudio posterior, determinó que la enseñanza abierta se vincula con el aprendizaje activo, manteniendo una cercanía a los estudios sociales (Chiang, Díaz \& Arriagada, 2016).

Dentro del análisis inferencial, se determinó que no se encuentra una relación funcional entre el estilo abierto con el rango de edades y el conocimiento académico de enseñanza universitaria. Además, no se encontró vínculo del estilo formal con los rangos de edades. Por lo contrario, sí se halló relación con el conocimiento académico de enseñanza universitaria. 
Tabla 3. Estilo formal y el conocimiento académico de docencia universitaria

\begin{tabular}{|c|c|c|c|c|}
\hline \multirow[t]{2}{*}{ E. Formal } & & \multicolumn{2}{|c|}{$\begin{array}{c}\text { Conocimiento académico } \\
\text { en docencia }\end{array}$} & \multirow[b]{2}{*}{ Total } \\
\hline & & No & Sí & \\
\hline \multirow[t]{2}{*}{ Muy baja } & $f$ & 2 & 3 & 5 \\
\hline & $\%$ & $6,5 \%$ & $3,6 \%$ & $4,4 \%$ \\
\hline \multirow[t]{2}{*}{ Baja } & $f$ & 8 & 18 & 26 \\
\hline & $\%$ & $25,8 \%$ & $21,7 \%$ & $22,8 \%$ \\
\hline \multirow[t]{2}{*}{ Moderada } & $f$ & 6 & 40 & 46 \\
\hline & $\%$ & $19,4 \%$ & $48,2 \%$ & $40,4 \%$ \\
\hline \multirow[t]{2}{*}{ Alta } & $f$ & 10 & 19 & 29 \\
\hline & $\%$ & $32,3 \%$ & $22,9 \%$ & $25,4 \%$ \\
\hline \multirow[t]{2}{*}{ Muy alta } & $f$ & 5 & 3 & 8 \\
\hline & $\%$ & $16,1 \%$ & $3,6 \%$ & $7,0 \%$ \\
\hline \multirow[t]{2}{*}{ Total } & $f$ & 31 & 83 & 114 \\
\hline & $\%$ & $100,0 \%$ & $100,0 \%$ & $100,0 \%$ \\
\hline$x^{2}$ & \multicolumn{3}{|c|}{11,049} & \\
\hline Df & \multicolumn{3}{|l|}{4} & \\
\hline$D$ & \multicolumn{3}{|l|}{ การ* } & \\
\hline
\end{tabular}

Según la tabla 3, se determinó que quienes poseían un conocimiento académico sobre docencia universitaria mantenían un nivel moderado $(48,2 \%)$ con tendencia alta en el estilo formal. Por otro lado, la tendencia alta correspondió a quienes no había recibido formación sobre docencia universitaria, manteniendo un porcentaje de $32,3 \%$. Según la tabla 3 , se determinó que quienes poseían un conocimiento académico sobre docencia universitaria mantenían un nivel moderado $(48,2 \%)$ con tendencia alta en el estilo formal. Por otro lado, la tendencia alta correspondió a quienes no había recibido formación sobre docencia universitaria, manteniendo un porcentaje de $32,3 \%$. Por lo que se colige que los docentes mantienen un nivel moderado $(40,4 \%)$ en el estilo formal. Este estilo se caracteriza por una docencia rígida y exigente, no acepta improvisaciones, y suele seguir el programa educativo del curso. Sin embargo, impulsan el análisis y reflexión, y permite que se sustenten las ideas de manera racional. No suele realizar trabajos en equipos, mantienen un nivel alto de responsabilidad y son cuidadosos (Collantes-Hidalgo, 2016). Este estilo suele relacionarse funcionalmente con el estilo teórico del estudiante.

Tabla 4. Estilo estructurado y el conocimiento académico de docencia universitaria.

\begin{tabular}{|c|c|c|c|c|}
\hline \multirow{2}{*}{$\begin{array}{c}\text { E. } \\
\text { Estructural }\end{array}$} & & \multicolumn{2}{|c|}{$\begin{array}{l}\text { Desarrollo académico } \\
\text { en docencia }\end{array}$} & \multirow[t]{2}{*}{ Total } \\
\hline & & No & Sí & \\
\hline \multirow[t]{2}{*}{ Muy baja } & $f$ & 1 & 16 & 17 \\
\hline & $\%$ & $3,2 \%$ & $19,3 \%$ & $14,9 \%$ \\
\hline \multirow[t]{2}{*}{ Baja } & $f$ & 4 & 21 & 25 \\
\hline & $\%$ & $12,9 \%$ & $25,3 \%$ & $21,9 \%$ \\
\hline \multirow[t]{2}{*}{ Moderada } & $f$ & 14 & 21 & 35 \\
\hline & $\%$ & $45,2 \%$ & $25,3 \%$ & $30,7 \%$ \\
\hline \multirow[t]{2}{*}{ Alta } & $f$ & 8 & 12 & 20 \\
\hline & $\%$ & $25,8 \%$ & $14,5 \%$ & $17,5 \%$ \\
\hline \multirow[t]{2}{*}{ Muy alta } & $f$ & 4 & 13 & 17 \\
\hline & $\%$ & $12,9 \%$ & $15,7 \%$ & $14,9 \%$ \\
\hline \multirow[t]{2}{*}{ Total } & $f$ & 31 & 83 & 114 \\
\hline & $\%$ & $100,0 \%$ & $100,0 \%$ & $100,0 \%$ \\
\hline$X^{2}$ & 10,153 & & & \\
\hline Df & 4 & & & \\
\hline $\mathrm{P}$ &, 038 & & & \\
\hline
\end{tabular}

En la tabla 4, el estilo Estructural demostró estar presente con nivel moderado y cierta inclinación a los niveles altos en aquellos docentes que no han llevado cursos ni especializaciones académicas en enseñanza. Por lo contrario, los docentes que sí han llevado estudios en enseñanza universitaria mantienen un tendencia moderada-baja, dado que los niveles moderados, bajos y muy baja suman un total de $69,9 \%$. Además, en la totalidad de docentes, el nivel moderado tiene un $30,7 \%$ de presencia en los docentes. El estilo estructurado se ve relacionado con el rango de edad de los docentes. Asimismo, (Martínez, 2009) aduce que existe influencia del estilo estructurado con los años de experiencia docente. 
Tabla 5. Estilo Funcional y el conocimiento académico de docencia universitaria

\begin{tabular}{|c|c|c|c|c|}
\hline \multirow[t]{2}{*}{$\begin{array}{c}\text { E. } \\
\text { Funcional }\end{array}$} & & \multicolumn{2}{|c|}{$\begin{array}{l}\text { Conocimiento } \\
\text { académico en } \\
\text { docencia }\end{array}$} & \multirow[t]{2}{*}{ Total } \\
\hline & & No & Sí & \\
\hline \multirow[t]{2}{*}{ Muy baja } & $f$ & 1 & 5 & 6 \\
\hline & $\%$ & $3,2 \%$ & $6,0 \%$ & $5,3 \%$ \\
\hline \multirow[t]{2}{*}{ Baja } & $f$ & 5 & 4 & 9 \\
\hline & $\%$ & $16,1 \%$ & $4,8 \%$ & $7,9 \%$ \\
\hline \multirow[t]{2}{*}{ Moderada } & $f$ & 19 & 35 & 54 \\
\hline & $\%$ & $61,3 \%$ & $42,2 \%$ & $47,4 \%$ \\
\hline \multirow[t]{2}{*}{ Alta } & $f$ & 5 & 21 & 26 \\
\hline & $\%$ & $16,1 \%$ & $25,3 \%$ & $22,8 \%$ \\
\hline \multirow[t]{2}{*}{ Muy alta } & $f$ & 1 & 18 & 19 \\
\hline & $\%$ & $3,2 \%$ & $21,7 \%$ & $16,7 \%$ \\
\hline \multirow[t]{2}{*}{ Total } & $f$ & 31 & 83 & 114 \\
\hline & $\%$ & $100,0 \%$ & $100, .0 \%$ & $100,0 \%$ \\
\hline $\mathrm{X}^{2}$ & \multicolumn{4}{|c|}{11,183} \\
\hline Df & \multicolumn{4}{|c|}{4} \\
\hline $\mathrm{P}$ & \multicolumn{4}{|l|}{025} \\
\hline
\end{tabular}

Como último resultado, la tabla 5 , demuestra que el estilo funcional predomina en el estilo moderado; para los docentes que no han recibido una instrucción sobre docencia universitaria se presenta con un $61,3 \%$, mientras que para los docentes que sí han recibido una instrucción se presenta en un 42,2\%. Finalmente, en su totalidad de docentes, el estilo Funcional predomina en el nivel moderado con un 47,4\%; y muestra tendencia a los niveles altos. Estos resultados permiten analizar que, gran cantidad de docentes mantienen tendencia alta en este estilo. Es decir, se caracterizan por una enseñanza con explicaciones breves, se emplea el sistema de reforzamiento en los éxitos, y su sistema se basa en la práctica (Collantes-Hidalgo, 2016). Suele ser uno de los preferidos por los docentes (Martínez, 2009). Por lo que, para este estilo existe amplia relación con los años de docencia (Renés, 2017a; Renés, 2017b) y con el desarrollo profesional del docente.

Por otro lado, es común que el docente universitario no prescinda de un solo estilo de ense- ñanza, dependerá del estudiante, la materia y el contenido. Suelen mostrar preferencia por diversos estilos de enseñanza, los cuales pueden vincularse con los estilos de aprendizaje (Ovierdo, Cárdenas, Zapata, Rendon \& Rojas, 2010). Estos estilos trascienden desde la planificación hasta el desarrollo del tema (Isaza \& Henao, 2012). Por tal motivo, Chiang, Díaz \& Rivas (2013) propone que el docente debe realizar un auto análisis previo desde la perspectiva del estudiante comprender su aprendizaje y modificar su estilo de enseñanza.

A pesar de las limitaciones, los hallazgos del presente estudio contribuyen a ampliar el conocimiento sobre la didáctica de los docentes convirtiendo en un antecedente para futuras investigaciones referido a los estilos de enseñanza en el nivel universitario.

\section{CONCLUSIONES}

La investigación basada en un marco de educación universitaria de pregrado demostró que no existe vínculo del estilo abierto con el factor estudiado. Los estilos estructurados y funcional son los que mantienen relaciones significativas con el conocimiento académico sobre docencia universitaria. Además, el estilo funcional es el de mayor elección por los docentes universitarios debido a la capacidad de adaptarse y de ejercer mayor práctica que teoría. Basado en un sistema por competencia mantiene lógica la preferencia por el estilo funcional. Finalmente, se determinó que los estilos de enseñanza no son características fijas en cada docente, sino que la adaptabilidad de los mismo, tomando en consideración ciertos aspectos como tiempo de clase, sílabo, materiales, temas y materia del curso, son fundamentales para tomar una postura determinada y modificar su estilo de enseñanza. 
REFERENCIAS BIBLIOGRÁFICAS

Aguilera, E. (2012). Los estilos de enseñanza, una necesidad para la atención de los estilos de aprendizaje en la educación universitaria. Revista Estilos de Aprendizaje, 10(5), 79-87.

Arias, J. L.; Morales, M.; Meroño, L.; \& Calderón, A. (2019). Enfoque de aprendizaje y rendimiento académico tras una intervención de carácter constructivista en estudiantes universitarios. Investigación e Innovación en la Enseñanza Superior: Nuevos contextos, nuevas ideas, 16-24.

Chiang, M. T.; Díaz, C.; \& Arriagada, P. (2016). Estilos de enseñanza y aprendizaje: ¿Cómo dialogan en la práctica? Revista Estilos de Aprendizaje, 9(7), 2-24.

Chiang, M. T.; Díaz, C.; \& Rivas, A. (2013). Un cuestionario de estilos de enseñanza para el docente de Educación Superior. Revista Lasallista de Investigación, 10(2), 62-68. Recuperado desde https:// www.redalyc.org/pdf/695/69529816008.pdf

Chiang, M. T.; Díaz, C.; Rivas, A.; \& Martínez, P. (2013). Validación del cuestionario estilos de enseñanza (cee). Un instrumento para el docente de educación superior. Revista Estilos de Aprendizaje, 11 (11), 1-18

Collantes-Hidalgo, J. (2016). Estilos de enseñanza de los docentes universitarios de la facultad de Educación de la Universidad Nacional Federico Villareal, Universidad Nacional Pedro Ruiz Gallo y del Departamento de Matemática de la Universidad Nacional Agraria La Molina (tesis de maestría). Universidad de Piura.

De León, I. J. (2006). Los estilos de en- señanza pedagógicos: Una propuesta de criterios para su determinación. Revista de Investigación, (57), 69-97

Delgado, M. (1996). Aplicaciones a los Estilos de Enseñanza en la Educación primaria. En C. Romero. Estrategias Metodológicas para el aprendizaje de los contenidos de Educación Física (73-86). Granada, España: Universidad de Granada.

Gómez, J. (2017). Nuevos estilos de enseñanza en la era de la convergencia tecno-mediática: hacia una educación holística e integral. International Journal Of Educational Research and Innovation, 8, 60-78. Recuperado desde https://rio.upo. es/xmlui/bitstream/handle/10433/5314/26018077-1-PB.pdf?sequence $=1 \&$ is Allowed $=y$

González-Peiteado, M. (2013). Los estilos de enseñanza y aprendizaje como soporte de la actividad docente. Revista de Estilos de Aprendizaje, 11(6), 51-70.

Hernández-Sampieri, R.; \& Mendoza, C. (2018). Metodología de la Investigación: La ruta cuantitativa, cualitativa $\mathrm{y}$ mixta. Ciudad de México, México: McGraw-Hill Interamericana Editores S.A.

Isaza, L.; \& Henao, G. (2012). Actitudes-Estilos de enseñanza: Su relación con el rendimiento académico. International Journal of Psychological Research, 5(1), 133-141. Recuperado desde https://www. redalyc.org/pdf/2990/299023539015.pdf

Lebrero-Casanova, I.; Almagro, B. J.; \& Sáenz-López, P. (2019). Estilos de enseñanza participativos en las clases de Educación Física y su influencia sobre diferentes aspectos psi- 
cológicos. Espiral Cuadernos del Profesorado: Revista Multidisciplinar de Educación, 12(25), 30-39. Recuperado desde http://repositorio. ual.es/bitstream/handle/10835/7296/22867916 - 1 - P B . pdf ? s e que n c e $=1$

Martínez, P. (2009). Estilos de enseñanza: Conceptualización e Investigación. Revista Estilos de Aprendizaje, 3(2).

Martínez, P. (2009). Estilos de enseñanza: conceptualización e investigación. Revista Estilos de Aprendizaje, 3(2), 3-19.

Ovierdo, P. E.; Cárdenas, F. A.; Zapata, P. N.; Rendon, M. \& Rojas, Y. A. (2010). Estilos de enseñanza y estilos de aprendizaje: implicaciones para la educación por ciclos. Actualidades Pedagógicas, (55), 31-43. Recuperado desde https://ciencia.lasalle.edu.co/ cgi/viewcontent.cgi?article $=1064 \&$ context $=$ ap

Rendón, M. A. (2013). Hacia una conceptualización de los estilos de enseñanza. Revisiones Colombiana de Educación, (64). Recuperado desde http://www. scielo.org.co/pdf/rcde/n64/n64a08.pdf

Renés, P. (2017a). Diagnóstico de los Estilos de Enseñanza del profesorado en España. Revista Electrónica de Educación Superior. Recuperado desde http://repositorio.unican.es:8080/ xmlui/bitstream/handle/10902/13642/DiagnosticoEstilosEnse $\%$ Flanza.pdf?sequence $=3$

Renés, P. (2017b). Estilos de Enseñanza: un referente para el nivel educativo de Formación Profesional. Universidad de Cantabria. Recuperado desde http://innovare.udec.cl/wp-content/uploads/2017/12/Art-4-tomo-3.pdf
Renés, P.; \& Martínez, P. (2016). Una mirada a los estilos de enseñanza en función de los estilos de aprendizaje. Revista de Estilos de Aprendizaje, 9(8), 224-243.

Renés, P.; Echeverry, L.; Chiang, M. T.; Rangel, L.; \& Geijo, P. (2013). Estilos de enseñanza: Un paso adelante en su conceptualización y diagnóstico. Revista Estilos de Aprendizaje, 11(6).

Rodríguez, N. E.; Delgadillo, M. D.; \& Torres, S. L. (2018). Los ambientes de aprendizaje constructivistas como alternativa para generar innovación en la universidad. International Journal of Information Sustems and Software Engineering for Big Companies (IJISEBC), 5(2), 41-52. Recuperado desde http://uajournals.com/ ojs/index.php/ijisebc/article/view/397/293

Sicilia, A. \& Delgado, M. (2002). Educación Física y Estilos de enseñanza. Barcelona, España: INDE. 